\title{
2D-QSAR, Docking Studies, and In Silico ADMET Prediction of Polyphenolic Acetates as Substrates for Protein Acetyltransferase Function of Glutamine Synthetase of Mycobacterium tuberculosis
}

\author{
Prija Ponnan, ${ }^{1,2}$ Shikhar Gupta, ${ }^{3}$ Madhu Chopra, ${ }^{4}$ Rashmi Tandon, ${ }^{1,2}$ \\ Anil S. Baghel, ${ }^{1}$ Garima Gupta, ${ }^{1}$ Ashok K. Prasad, ${ }^{2}$ Ramesh C. Rastogi, \\ Mridula Bose, ${ }^{1}$ and Hanumantharao G. Raj ${ }^{1}$ \\ ${ }^{1}$ Department of Biochemistry and Microbiology, V. P. Chest Institute, University of Delhi, Delhi 110 007, New Delhi, India \\ ${ }^{2}$ Department of Chemistry, University of Delhi, Delhi 110 007, New Delhi, India \\ ${ }^{3}$ Department for Pharmacoinformatics, National Institute of Pharmaceutical Education and Research, S.A.S. Nagar, Mohali, \\ Punjab 160062, India \\ ${ }^{4}$ Dr. B.R. Ambedakar Centre for Biomedical Research, University of Delhi, Delhi 110 007, India
}

Correspondence should be addressed to Shikhar Gupta; shiksun@gmail.com

Received 30 November 2012; Accepted 20 December 2012

Academic Editors: M. Espinoza-Fonseca and D. D. Leonidas

Copyright (C) 2013 Prija Ponnan et al. This is an open access article distributed under the Creative Commons Attribution License, which permits unrestricted use, distribution, and reproduction in any medium, provided the original work is properly cited.

A novel transacetylase (TAase) function of glutamine synthetase (GS) in bacterial species such as Mycobacterium smegmatis and Mycobacterium tuberculosis H37Rv was established by us, termed as mycobacterial TAase (MTAase). Several polyphenolic acetates (PAs) were found to be substrates for MTAase by inhibiting certain receptor proteins such as glutathione S-transferase by way of acetylation. The present work describes the descriptor-based 2D-QSAR studies developed for a series of PA synthesized by us and evaluated for MTAase and antimycobacterial activity using stepwise multiple linear regression method with the kinetic constants and the minimum inhibitory constant (MIC) as the dependent variables, to address the fact that TAase activity was leading to the antimycobacterial activity. Further, blind docking methods using AutoDock were carried out to study the interaction of potent PA with the crystal structure of $M$. tuberculosis GS. PAs were predicted to bind $M$. tuberculosis GS on the protein surface away from the known active site of GS. Subsequent focussed/refined docking of potent PA with GS showed that the $\varepsilon$-amino group of Lys4 of GS formed a cation- $\pi$ interaction with the benzene ring of PA. Also, ADMET-related descriptors were calculated to predict the pharmacokinetic properties for the selection of the effective and bioavailable compounds.

\section{Introduction}

Our laboratory is credited for the discovery of novel TAase which catalyzes the possible transfer of acetyl group from PA to certain functional proteins such as GST, cytochrome P-450 reductase, and nitric oxide synthase (NOS) leading to their functional modifications [1-3]. An assay procedure was developed utilizing the inhibition of cytosolic GST brought about by TAase-catalyzed acetylation by PA. Both the substrates, namely, the target protein GST and the acetyl group donor PAs were found to take part in the TAasecatalyzed bimolecular reaction [2]. This assay procedure was utilized to purify TAase from tissues like human placenta and rat liver and characterized as calreticulin, a calciumbinding ER luminal protein $[4,5]$. The acetylation of receptor proteins such as GST and NOS at $\varepsilon$-amino group lysine residues was established by immunoblotting using acetylated lysine antibody and mass spectrometry [6,7]. Recently, TAase was identified and established by us in bacterial species such as Mycobacterium smegmatis [8] and Mycobacterium 
tuberculosis (Mtb) H37Rv [9] as glutamine synthetase (GS). Glutamine synthetase catalyzes the conversion of glutamate to glutamine in the presence of ammonium ion with simultaneous hydrolysis of ATP which is used as the energy source and plays an essential role in bacterial nitrogen metabolism $[10,11]$. Several PAs, including acetoxycoumarins in general, were found to be the substrates for mycobacterial TAase (MTAase). The specificities of various acetoxycoumarins towards MTAase were determined by their ability to inhibit GST irreversibly, and their kinetic constants $\left(K_{m}\right.$ and $\left.V_{\max }\right)$ were determined [9]. Several inhibitors are known for GS and most of them are analogues of glutamate and replace this substrate in the active site of the enzyme. Among the known inhibitors methionine sulfoximine (MSO) and 2amino-4-(hydroxymethyl-phosphoryl) butanoic acid (phosphinothricin) are the well-established inhibitors of GS [12, 13]. During the examination of the role of GS inhibitor on MTAase function of GS, it was observed that MSO failed to inhibit MTAase-catalysed reaction, indicating that the TAase activity of MTAase is independent of the catalytic activity of GS $[8,9]$. Electron microscopic studies carried out by us have shown cell wall attacking properties of these compounds in M. smegmatis [8] and M. tuberculosis [14]. The cell wall of Mycobacterium species is responsible for maintaining the cell integrity and thus is considered to be a potential drug target owing to its crucial role in cell survival and viability. If a compound is found to affect the cell wall or its biosynthesis in any manner, it is bound to bring about the inhibition of bacterial growth. The PAs referred to in the present study have been reported earlier [14] to possess "cell-wall attacking" characteristic; that is, these molecules have been found to bring about changes in cell morphology ranging from indentations in the wall to complete rupturing of cell wall along with extrusion of cytoplasmic material in some cases and complete disintegration/disappearance of the wall in others [14]. Moreover, these pronounced changes were recorded when the bacteria were grown in the presence of sublethal doses of the test molecules. These observations led us to believe that these compounds may serve as potential drug candidates, and, therefore, these were further explored to determine their drug likeness and also establish a structure activity relationship [14]. The present work describes the descriptor-based QSAR studies developed for a series of acetoxycoumarins synthesized by us and evaluated for TAase and antimycobacterial activity. Also, ADMET-related descriptors were calculated to predict the pharmacokinetic properties for the selection of the effective and bioavailable compounds. Further, docking studies were done to analyze the interaction of the potent acetoxycoumarins with the crystal structure of M. tuberculosis GS.

\section{Methodology}

\subsection{D-QSAR Analysis}

2.1.1. Data Set and Methodology. Compounds 1-14 were synthesized and characterized following the published synthetic procedures $[1-3,9,15]$. The PAs were screened for their antimycobacterial activity and TAase activity that are listed in Table 1 according to our published methods [8], utilizing Mtb GS as the target protein. Biological activity data, reported as MIC values for the antimycobacterial activity and kinetic constants $\left(K_{m}\right.$ and $\left.V_{\max }\right)$ for TAase activity (Table 1$)$, were first converted to - $\log$ MIC on molar basis and $\log \left(V_{\max } / K_{m}\right)$, respectively, and were used as the dependent variables to get the linear relationship in the QSAR models.

Hyperchem-8 program [16] was used to build the structures and perform geometry optimizations of the compounds. The lowest energy conformations of the compounds were determined first by minimizing the structures by molecular mechanics method using MM+ force field followed by semiempirical self-consistent field molecular orbital (SCF MO) theory (parametric model 3 (PM3) method within the restricted Hartree-Fock (RHF) formalism). Conjugate gradient method (Polak-Ribiere algorithm) with SCF convergency set to $0.001 \mathrm{kcal} / \mathrm{mol}$ was considered in the geometry optimization stage of calculations. Frequency calculations have been performed to confirm all stationary points.

2.1.2. Molecular Descriptors for QSAR Analysis. TSAR 3.3 software package (Accelrys, San Diego, CA, USA) was employed to calculate descriptors for entire molecule and the defined substituents. Substituents were defined for all PA, a single hydrogen atom also served as a substituent (Table 1). TSAR includes various physicochemical, topological, and electrostatic descriptors: molecular surface area and volume, molecular mass, moments of inertia (moment 1 , 2, 3 (size, length)), ellipsoidal volume, Verloop parameters, Dipole moments (total, bond and $\mathrm{x}, \mathrm{y}, \mathrm{z}$ components), Lipole moments (total, bond and $\mathrm{x}, \mathrm{y}, \mathrm{z}$ components), topological indices (Wiener, Randić, and Balaban indices), molecular connectivity indices ( $\mathrm{Chi}, \mathrm{ChiV}$ indices) of atoms, bonds, path, cluster and path/cluster, Molecular shape indices (Kappa, KAlpha indices), Electrotopological state indices, LogP, Atom counts (C,N,S \& H), Ring count (aromatic and aliphatic), and Group count (methyl, hydroxyl, ethyl), Electrostatic properties like Total energy, Electronic energy, Nuclear repulsion energy, Accessible surface area, Atomic charge, Mean polarizability, Heat of formation, HOMO and LUMO eigenvalues, Ionization potential, Total dipole, Polarizability, and Dipole components. Pairwise correlation analysis of the descriptors was performed, and the intercorrelated descriptors $(>0.6)$ were discarded depending on their individual correlation with the biological activity.

2.1.3. Stepwise Multiple Regression. In an effort to investigate the role of structural parameters, which appears to influence the observed activities of reported compounds, stepwise multiple linear regressions were performed using TSAR 3.3 software. TSAR uses a two-way stepping algorithm to select variables for the regression equation. At each step, partial $F$ values are calculated for each variable, as an estimate of their potential contribution to the model. The partial $F$ values are compared with the $F$-to- Leave and $F$-to-Enter settings. The overall $F$ statistic for a model is

$$
F=\frac{\text { explained mean square }}{\text { residual mean square }} .
$$


TABLE 1: Structures of PA used in the 2D-QSAR analysis with corresponding TAase and antimycobacterial activities.

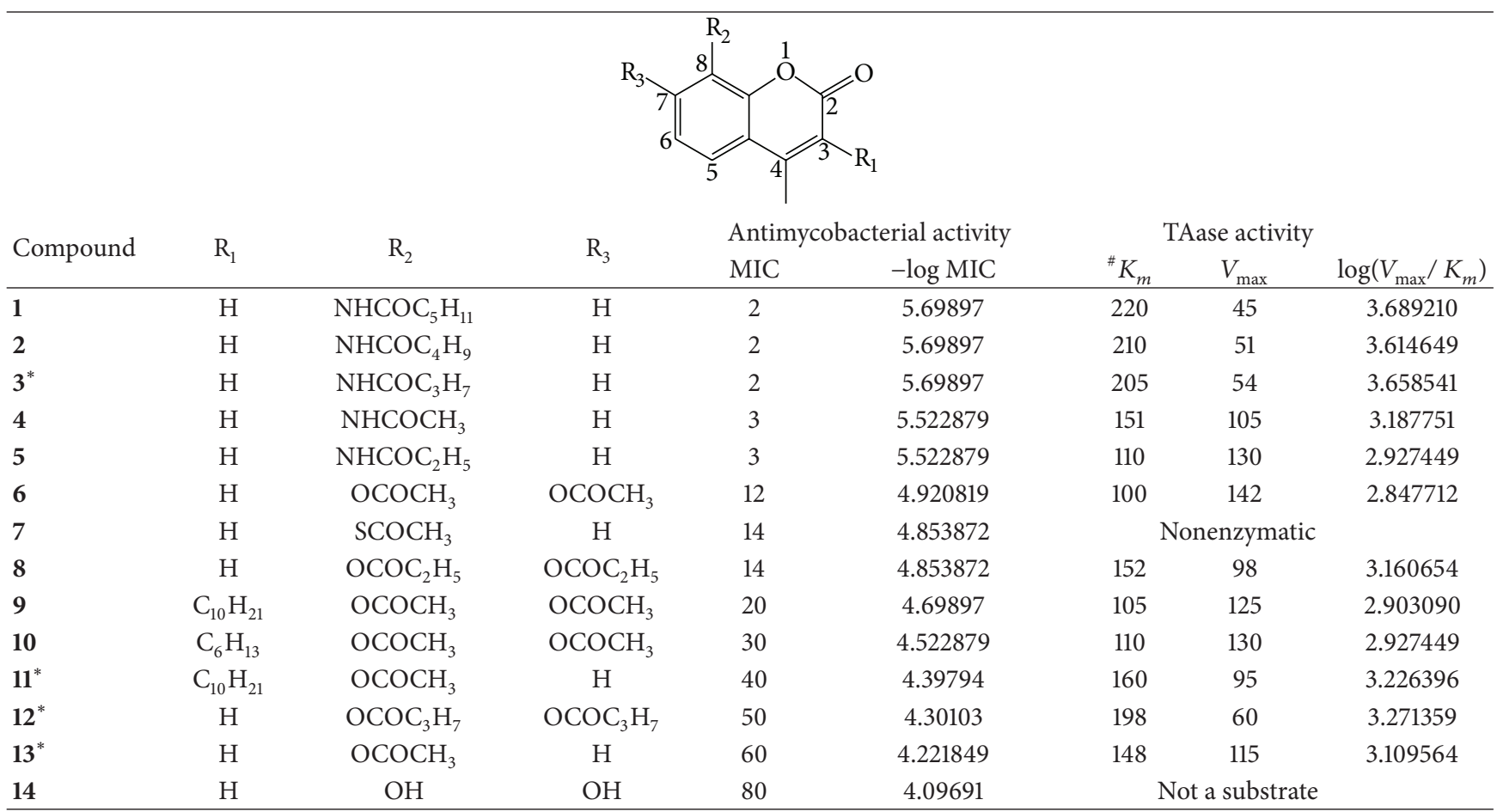

* Test set.

"Values are mean of three observations in triplicate with variation less than $5 \%$.

Partial $F$ values are an estimation of the sequential contribution towards the $F$ statistic for the final model. $F$ to-Leave: forward and backward stepping algorithms can give regression equations that use different variables. This is caused by collinearity or multicollinearity of variables in the data set and may indicate instability in the model. In a forward stepping process, once a variable has entered the model, it cannot leave. If $F$-to-Leave is set to zero, a forward stepping process is used. At each step, the partial Fvalues of all variables outside the model are calculated. If any variable has a value greater than $F$-to-Enter, the variable with the highest partial $F$ value is added to the model. The process is continued until no more variables qualify to enter the model, or the required number of steps has been reached. In a backward stepping process, all variables are used in the initial model (overriding any choice of starting variables). Once a variable has left the model, it may not reenter. If $F$-to-Enter is set to zero, a backward stepping process is used. At each step, the partial $F$ values of all variables inside the model are calculated. If any variable has a value less than $F$-to-Leave, the variable with the lowest partial $F$ value is removed from the model. The process is continued until no more variables qualify to leave the model, or the required number of steps has been reached.

The default values for "stepping," that is, F-to-Enter and $F$-to-Leave were set to 4 and 3.5, respectively. The whole dataset was randomly divided into test set (including compounds $3, \mathbf{1 1}, \mathbf{1 2}$, and $\mathbf{1 3}$ ) and remaining compounds as training set. Statistical quality of the regression models was judged based on parameters such as correlation coefficient $(r)$, squared correlation coefficient $\left(r^{2}\right)$, standard error of estimate $(s)$, and fisher test value ( $F$-value). A compound was considered as an outlier when the residual value exceeded 1.5 times the standard error of estimate in an equation. Further, the predictive ability of the model was quantified internally by determining cross-validated $r^{2}$ by leave-one-out (LOO) method $\left(\mathrm{q}_{\mathrm{LOO}}^{2}\right)$ and the predictive residual sum of squares (PRESS). Predictive ability of the generated model was validated by using the external test set by determining external set cross validation $r^{2}\left(q_{\text {ext }}^{2}\right)$, determination coefficient between observed and predicted values with $\left(r_{\text {pred }}^{2}\right)$ and without intercept $\left(r_{0}^{2}\right)$, slopes $k$ and $k^{\prime}$ of regressions through the origin of predicted versus observed and observed versus predicted intensities respectively. Models were considered to have high predictive ability $[17,18]$ if $q_{\text {ext }}^{2}>0.5, r_{\text {pred }}^{2}>0.6$, both $r_{0}^{2}$ and $r_{0}^{\prime 2}$ had to be close to each other such that $\left(r_{\text {pred }}^{2}-\right.$ $\left.r_{0}^{2}\right) / r_{\text {pred }}^{2}<0.1$ or $\left(r_{\text {pred }}^{2}-r_{0}^{\prime 2}\right) / r_{\text {pred }}^{2}<0.1$, and the corresponding slopes should follow the criteria $0.85 \leq k \leq 1.15$ or $0.85 \leq k^{\prime} \leq 1.15[17,18]$.

2.1.4. ADMET Prediction for Acetoxycoumarins. Absorption, distribution, metabolism, elimination, and toxicity (ADMET) properties were predicted using ADMET descriptors in Discovery Studio 2.1 (Accelrys, San Diego, CA, USA). The module uses six mathematical models, to quantitatively predict properties by a set of rules/keys (Table 2) that specify threshold ADMET characteristics for the chemical structure of the molecules based on the available drug 
TABLE 2: ADMET descriptors and their rules/keys.

ADMET absorption level (human intestinal absorption)

\begin{tabular}{lc} 
Level & Description \\
\hline 0 & Good absorption \\
1 & Moderate absorption \\
2 & Low absorption \\
3 & Very low absorption
\end{tabular}

\begin{tabular}{|c|c|c|}
\hline \multicolumn{3}{|c|}{ ADMET aqueous solubility level } \\
\hline Level & Value & Description \\
\hline 0 & $\begin{array}{c}\log (\text { molar solubility }) \\
<-8.0\end{array}$ & Extremely low \\
\hline 1 & $\begin{array}{l}-8.0<\log (\text { molar } \\
\text { solubility })<-6.0\end{array}$ & No, very low, but possible \\
\hline 2 & $\begin{array}{l}-6.0<\log (\text { molar } \\
\text { solubility })<-4.0\end{array}$ & Yes, low \\
\hline 3 & $\begin{array}{l}-4.0<\log (\text { molar } \\
\text { solubility })<-2.0\end{array}$ & Yes, good \\
\hline 4 & $\begin{array}{c}-2.0<\log (\text { molar } \\
\text { solubility })<0.0\end{array}$ & Yes, optimal \\
\hline 5 & $0.0<\log$ (molar solubility) & No, too soluble \\
\hline 6 & -1000 & $\begin{array}{c}\text { Warning: molecules with } \\
\text { one or more unknown } \\
\text { AlogP98 types }\end{array}$ \\
\hline
\end{tabular}

ADMET (blood brain barrier penetration level) BBB

\begin{tabular}{lc} 
Level & Description \\
\hline 0 & Very High \\
1 & High \\
2 & Medium \\
3 & Low \\
4 & Undefined \\
& Warning: molecules with \\
5 & one or more unknown \\
& AlogP calculation \\
\hline
\end{tabular}

\begin{tabular}{lc}
$\begin{array}{l}\text { Predicted } \\
\text { class }\end{array}$ & Value \\
\hline 0 & $\begin{array}{c}\text { Noninhibitor } \\
\text { Inhibitor }\end{array}$ \\
\hline & ADMET hepatotoxicity \\
Predicted & \\
class & Value \\
\hline 0 & Nontoxic \\
1 & Toxic \\
\hline ADMET (plasma protein binding level) PPB & \\
Level & Description \\
\hline 0 & Binding is $<90 \%$ \\
1 & Binding is $\geq 90 \%$ \\
2 & Binding is $\geq 95 \%$ \\
\hline
\end{tabular}

information: ADMET absorption predicts human intestinal absorption (HIA) after oral administration. The model was developed using 199 compounds in the training set based on the calculations AlogP (ADMET_AlogP98) and 2D polar surface area (PSA_2D). The absorption levels of HIA model are defined by $95 \%$ and $99 \%$ confidence ellipses in the ADMET_PSA_2D, ADMET_AlogP98 plane [19]. These ellipses describe the regions where well-absorbed compounds are expected to be found. The upper limit of PSA_2D value for the $95 \%$ confidence ellipsoid is at 131.62, while the upper limit of PSA_2D value for the $99 \%$ confidence ellipsoid is at 148.12. ADMET aqueous solubility predicts the solubility of each compound in water at $25^{\circ} \mathrm{C}$. The model is based on genetic partial least squares method on a training set of 784 compounds with experimentally measured solubilities [20]. ADMET blood brain barrier model predicts bloodbrain penetration (blood brain barrier, BBB) of a molecule after oral administration. This model was derived from a quantitative linear regression model for the prediction of blood-brain penetration, as well as $95 \%$ and $99 \%$ confidence ellipses (analogous to that of HIA) in the ADMET_PSA_2D, ADMET_AlogP98 plane. They were derived from over 800 compounds that are known to enter the CNS after oral administration [21]. ADMET plasma protein binding model predicts whether a compound is likely to be highly bound to carrier proteins in the blood. Predictions are based on AlogP98 and 1D similarities to two sets of "marker" molecules. One set of markers is used to flag binding at a level of $90 \%$ or greater, and the other set is used to flag binding at a level of $95 \%$ or greater. Binding levels predicted by the marker similarities are modified according to conditions on calculated $\log \mathrm{P}$ [22]. ADMET CYP2D6 binding predicts cytochrome P450 2D6 enzyme inhibition using 2D chemical structure as input as well as a probability estimate for the prediction. Predictions are based on a training set of 100 compounds with known CYP2D6 inhibitions [23]. ADMET hepatotoxicity predicts the potential human hepatotoxicity for a wide range of structurally diverse compounds. Predictions are based on an ensemble recursive partitioning model of 382 training compounds known to exhibit liver toxicity (i.e., positive dose-dependent hepatocellular, cholestatic, neoplastic, etc.) or to trigger dose-related elevated aminotransferase levels in more than 10 percent of the human population [24].

2.1.5. Molecular Docking. In order to corroborate the novel TAase function of Mtb GS, it was thought important to study the interaction of model PA, 7,8-diacetoxy-4methylcoumarin (DAMC), 7-acetoxy-4-methylcoumarin (7$\mathrm{AMC}$ ), and 7-NH-acetoxy-4-methylcoumarin (7-NH-AMC) with the structure of Mtb GS using computational docking study. In the absence of any known active site for the TAase activity of Mtb GS, blind docking approach was utilized wherein the entire protein surface is scanned for the probable ligand binding sites for PA [25]. For this purpose Autodock program was used [26] and PAs were docked to the crystal structure of Mtb GS (PDB ID: 2BVC) [27] in two steps. Firstly, a grid field of $60 \AA$ cube with grid points separated by $1 \AA$ centered at the middle of the protein was considered using AUTOGRID. The final binding mode conformation was determined by focused/refined docking, where the binding site determined with blind docking was subjected to more detailed calculations by considering the 
TABLE 3: Descriptors included in the best model obtained for antimycobacterial and TAase activity.

\begin{tabular}{|c|c|c|c|c|c|c|}
\hline & Descriptor & Coefficient $^{\mathrm{a}}$ & Jackknife SE ${ }^{\mathrm{b}}$ & Covariance $\mathrm{SE}^{\mathrm{c}}$ & $t$-value ${ }^{\mathrm{d}}$ & $t$-probability ${ }^{\mathrm{e}}$ \\
\hline \multirow{4}{*}{$\begin{array}{l}\text { Antimycobacterial } \\
\text { activity }\end{array}$} & $\begin{array}{c}\text { X1: Balaban } \\
\text { topological index } \\
\text { (Substituent 2) }\end{array}$ & 0.25917 & 0.12484 & 0.050123 & 5.1706 & 0.0020731 \\
\hline & 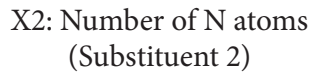 & 0.84199 & 0.10326 & 0.07821 & 10.766 & $3.7971 e-005$ \\
\hline & $\begin{array}{l}\text { X3: quadrupole } \\
\text { XX component } \\
\text { (whole molecule) }\end{array}$ & 0.064479 & 0.028036 & 0.032179 & 2.0037 & 0.091947 \\
\hline & C: constant & 4.0866 & 0.43577 & & & \\
\hline \multirow[t]{2}{*}{ MTAase activity } & $\begin{array}{c}\text { X1: balaban } \\
\text { topological index } \\
\text { (Substituent 2) }\end{array}$ & 0.13387 & 0.018757 & 0.027883 & 4.8012 & 0.0007223 \\
\hline & C: constant & 2.8493 & 0.045981 & & & \\
\hline
\end{tabular}

a The regressions coefficient for each variable in the QSAR equations. ${ }^{b}$ An estimate of the standard error on each regression coefficient derived from a jack knife method on the final regression model. ${ }^{\mathrm{c}}$ An estimate of the standard error on each regression coefficient derived from covariance matrix. ${ }^{\mathrm{d}}$ Measures the significance of each variable included in the final model.

estatistical significance for $t$ values.

grid field of $60 \AA$ cube, and the grid points were separated by $0.375 \AA$ centered on the best scored conformation obtained in the first step. Polar hydrogens and partial charges for proteins and ligands were added using the Kollman United atom and Gasteiger charges, respectively, using AUTODOCKTOOLS [28]. An automated molecular docking was performed using the hybrid genetic algorithm-local search (GA-LS). Default parameters were used for the number of generations, energy evaluations, and docking runs, which were set to 1,000 ; $25,000,000$ and 256 , respectively. The docking energy represents the sum of the intermolecular energy and the internal energy of the ligand while the free-binding energy is the sum of the intermolecular energy and the torsional-free energy [29].

\section{Results and Discussion}

3.1. QSAR Analysis. In an attempt to determine the role of structural features of PA, which appears to influence the antimycobacterial activity by its acyl group donating ability mediated by TAase, QSAR models was generated. The inhibitory activity of PA determined in terms of MIC values were taken as - log MIC and the logarithmic value of catalytic efficiency of PA $\left(\log \left(V_{\max } / K_{m}\right)\right)$ to donate acetyl group to receptor protein mediated by TAase were used as the dependent values in the QSAR study (Table 1). As indicated in Table 1 only 12 PAs were considered for TAase activity, compounds 7 being a nonenzymatic substrate whereby this compound is capable of acetylating receptor proteins independent of acetyltransferase and compound $\mathbf{1 4}$ which is the dihydroxy analogue of compound $\mathbf{6}$. The compound possesses hydroxyl group at C-7 and C-8 position and lacks acetyl group substituent and thus is a nonsubstrate for the protein acetyltransferase activity. Hence, these two compounds (compounds 7 and 14) were thus excluded from the QSAR model generation of TAase activity.

The QSAR model with high statistical significance, obtained for antimycobacterial activity can be represented by the following equation and the descriptors are detailed in Table 3:

$$
\begin{gathered}
-\log \text { MIC }=0.17540908 * \mathrm{X} 1+1.0271472 * \mathrm{X} 2 \\
+0.10474976 * \mathrm{X} 3+4.107533 \\
s=0.18, \quad F=41.94, \quad r=0.96, \quad r^{2}=0.93, \\
q_{\mathrm{LOO}}^{2}=0.77, \quad \text { PRESS }=1.04 .
\end{gathered}
$$

High predictive power of this model is demonstrated in Figure 1(a) and the histogram for residual is shown in Figure 1(b).

The obtained correlation equation was screened by using test set. Figures 2(a) and 2(b) illustrate the predictive ability of the QSAR, where the statistical parameters $r_{\text {pred }}^{2}=0.957$, $q_{\text {ext }}^{2}=0.88,\left(r_{\text {pred }}^{2}-r_{0}^{2}\right) / r_{\text {pred }}^{2}=0.071,\left(r_{\text {pred }}^{2}-r_{0}^{\prime 2}\right) / r_{\text {pred }}^{2}<$ $0.031, k=1.026, k^{\prime}=0.97$ were within the limits $[17,18]$.

The stepwise regression resulted in the following statistically significant monoparametric model for TAase activity and the details of the descriptor are provided in Table 3:

$$
\begin{gathered}
\log \left(V_{\max } / K_{m}\right)=0.13387173 * \mathrm{X} 1+2.8492985 \\
s=0.173, \quad F=23.05, \quad r=0.835, \\
r^{2}=0.697, \quad q_{\text {LOO }}^{2}=0.609, \quad \text { PRESS }=0.387 .
\end{gathered}
$$

The plot of the calculated versus predicted $\log \left(V_{\max } / K_{m}\right)$ is presented in Figure 3(a) and the histogram for residual is shown in Figure 3(b). 


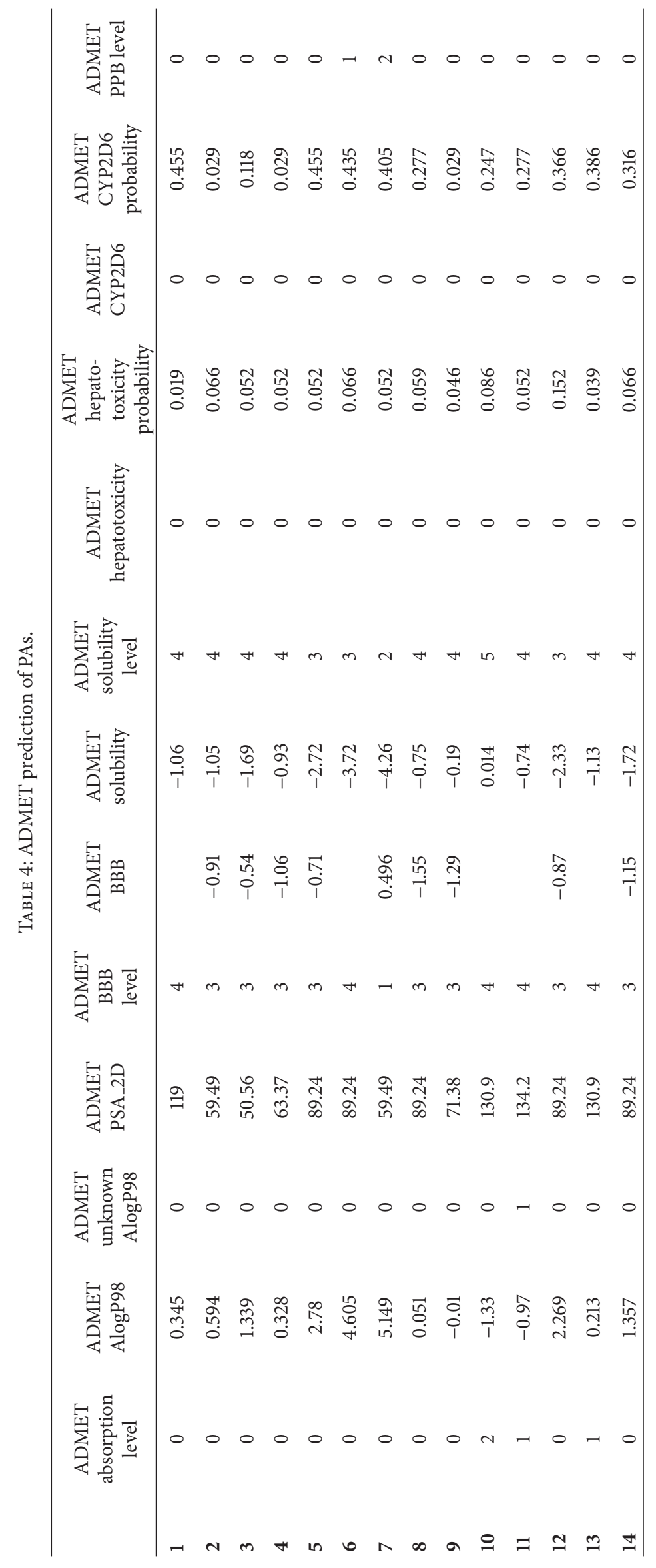




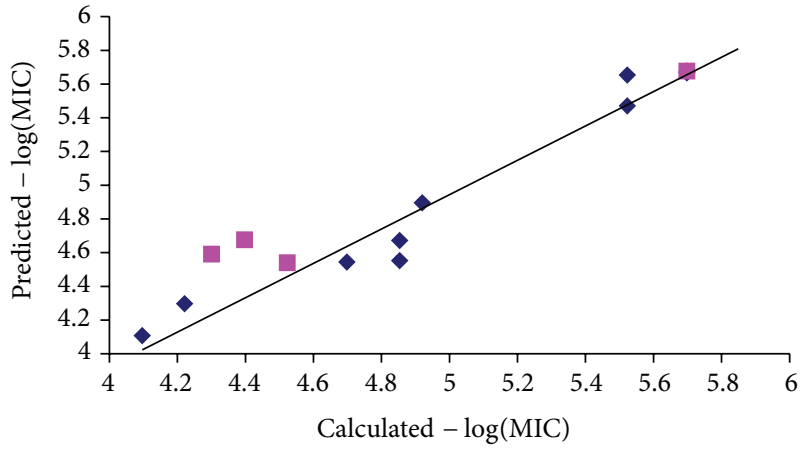

Training set

Test set

(a)

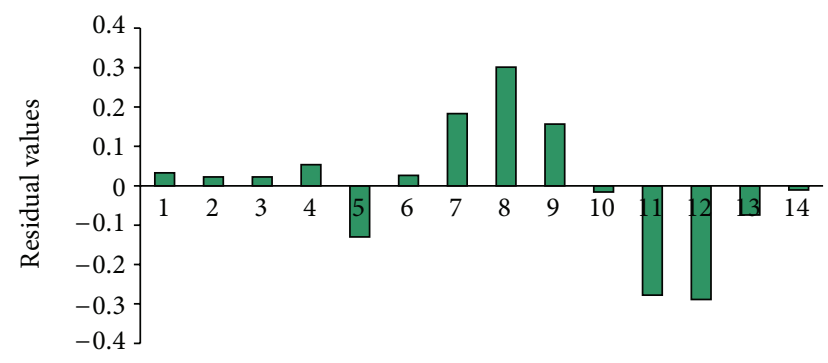

(b)

FIGURE 1: (a) Graph of calculated versus predicted - logMIC activities from QSAR model. (b) Histogram of residuals of calculated and predicted - logMIC activities PA in the training set.

The model also followed the criteria for the predictive ability of the QSAR (Figures 4(a) and 4(b)), and the statistical parameters, $r_{\text {pred }}^{2}=0.978, q_{\text {ext }}^{2}=0.603,\left(r_{\text {pred }}^{2}-r_{0}^{2}\right) / r_{\text {pred }}^{2}=$ $0.078,\left(r_{\text {pred }}^{2}-r_{0}^{\prime 2}\right) / r_{\text {pred }}^{2}<0.091, k=0.97, k^{\prime}=1.02$ were within the limits $[17,18]$.

The descriptors based on the model used in the present study are indicated in Table 3 . It is observed that all the descriptors have positive contribution to the antimycobacterial activity. The obtained QSAR model for antimycobacterial activity demonstrates the significance of Balaban index for substituent 2 of PA. The descriptor Balaban index is a type of topological index that represents extended connectivity and is a good descriptor for the shape of the molecules [31]. All the topological indices used are calculated from the hydrogensuppressed molecular graphs. Balaban index can be described as the average distance sum connectivity. Balaban index, $J$, of a connected molecular graph, $G$, can be defined as

$$
J(G)=\frac{E}{\mu+1} \sum_{\text {edges }}\left(d s_{i} d s_{j}\right)^{-1 / 2}
$$

where $E$ is the number of edges in $G$ and $\mu$ is the cyclomatic number of $G$. The cyclomatic number $\mu$ of a cyclic graph $G$ is equal to the minimum number of edges that must be removed before $G$ becomes acyclic and $d s_{i}(i=1,2, \ldots, N ; N$

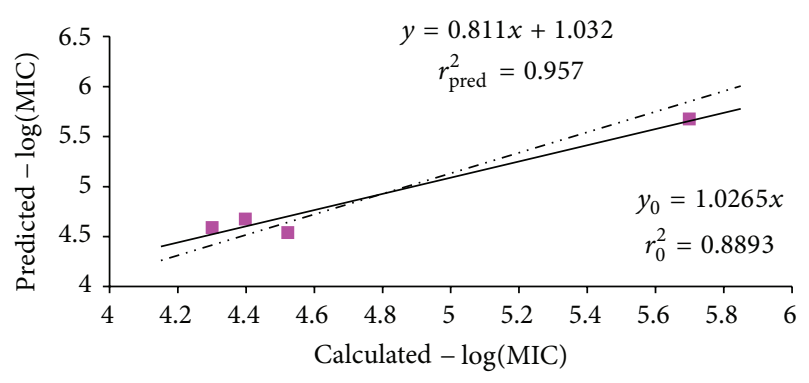

(a)

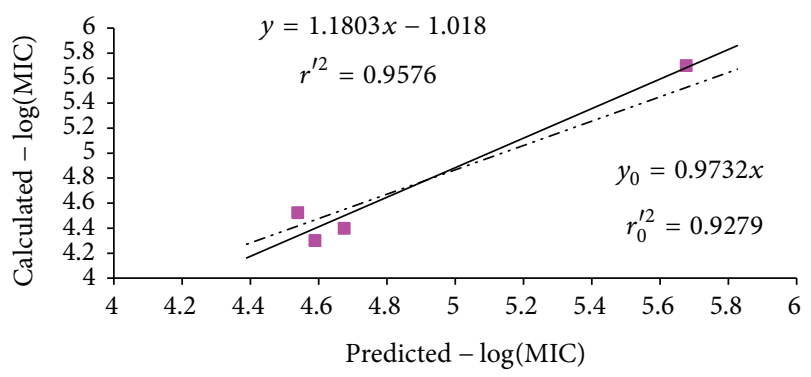

(b)

FIGURE 2: Regression plot between (a) calculated versus predicted values $(-\log \mathrm{MIC})$. The dotted line indicates the regression line through origin (for equation $y_{0}=1.0265 x$, with intercept $=0$ ), and the solid line indicates the regression lines for equation $y=0.811 x+$ 1.032 (with intercept $=1.032$ ) and $(b)$ predicted versus calculated values $\left(\log V_{\max } / K_{m}\right)$ for compounds from test set justifying the predictive ability of QSAR model. The dotted line indicates the regression line through origin (for equation $y_{0}=0.9732 x$, with intercept $=0)$, and the solid line indicates the regression lines for equation $y=1.1803 x-1.018$ (with intercept $=\neg 1.018$ ).

is the number of vertices in $G$ ) is a distance sum. The distance sum, $d s_{i}$, for a vertex $i$ represents the sum of all entries in the corresponding row (or column) of the distance matrix $D$ :

$$
d s_{i}=\sum_{j=1}^{N} D_{i j}
$$

The direct relationship between Balaban index of substituent at 2 nd position (C-7 position of coumarin ring) and -log MIC (see (2), Table 3) indicates that a bigger size and high branching of substituent 2 increase the antimycobacterial activity. Balaban index has been successfully used to study the antibacterial activity of sulfa drugs [32]. Similarly, the positive correlation coefficient for number of nitrogen atoms at substituent 2 shows the significance of $\mathrm{N}$-acyl substitution at 2nd position in PA (see (2), Table 3). The presence of this descriptor in high magnitude in (2) demonstrates the dominating role of $\mathrm{N}$-acyl substituted PA in antimycobacterial activity. The equation also expresses the significance of quadrupole XX component (whole molecule) for the antimycobacterial activity. It characterizes molecular charge distribution in PA. However, only Balaban topological index 


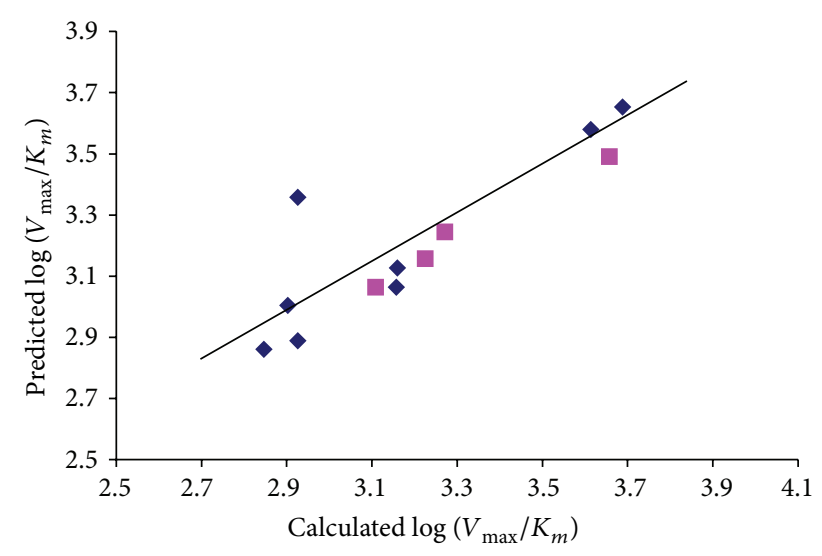

- Training set

Test set

(a)

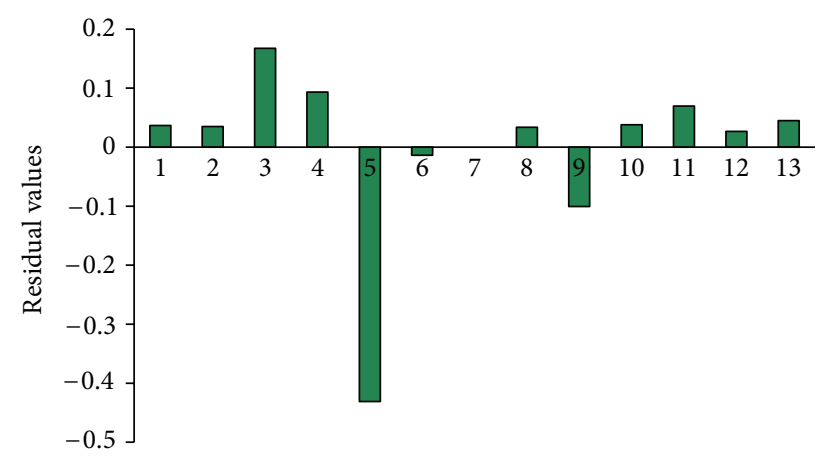

(b)

Figure 3: (a) Graph of calculated versus predicted $\log \left(V_{\max } / K_{m}\right)$ activities from QSAR model. (b) Histogram of residuals of calculated and predicted $\log \left(V_{\max } / K_{m}\right)$ activities PA in the training set.

for the substituent 2 of acetoxycoumarins showed significant correlation with the TAase activity (Table 3 ). Thus PA with high degree of bonding linearity with groups that increase molecular weight was found to possess TAase activity. Earlier, Basak et al. have indicated a predominant role of topological steric parameters such as connectivity indices and information theoretic topological indices in determining the rates of the enzymatic N-acetylation reaction [33]. Further, the significance of the descriptor Balaban topological index at substituent 2 could be understood in the way that PA with long-chain acyl group could be a good substrate for MTAase activity. This can be correlated with our recent investigations that led to the conclusion that PA with higher acyl group substituent at C-7 position (other than acetyl group) such 7propoxycoumarin was capable of transferring propoxy group to the receptor proteins [34]. Hence, MTAase could be viewed as accommodating PA with long chain acyl group in its active site. Other acetyltransferases such as histone acetyltransferase was found capable of accommodating higher chain CoAs (such as propionyl CoA and butyryl CoA) without steric hindrance [35]. These observations give a tacit explanation for

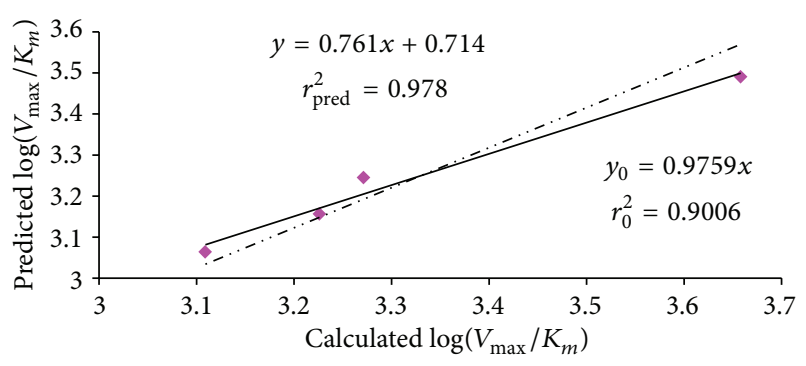

(a)

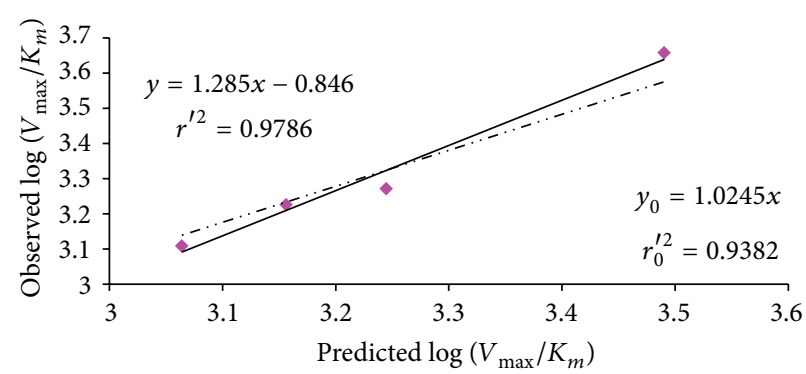

(b)

FIGURE 4: Regression plot between (a) calculated versus predicted values $\left(\log V_{\max } / K_{m}\right)$. The dotted line indicates the regression line through origin (for equation $y_{0}=0.9759 x$, with intercept $=0$ ), and the solid line indicates the regression lines for equation $y=0.761 x+$ 0.714 (with intercept $=0.714$ ) and $(b)$ predicted versus calculated values $\left(\log V_{\max } / K_{m}\right)$ for compounds from test set justifying the predictive ability of QSAR model. The dotted line indicates the regression line through origin (for equation $y_{0}=1.0245 x$, with intercept $=0)$, and the solid line indicates the regression lines for equation $y=1.25 x-0.846$ (with intercept $=\neg 0.846$ ).

the monoparametric model (3) for TAase activity. Furthermore, it is important to note the occurrence of an overlapping descriptor (Balaban topological index at substituent 2) from our two QSAR models, clearly indicates that TAase activity mediated by GS utilizing PA as acetoxy group donor was leading to the antimycobacterial activity of PA.

3.2. Binding Studies. Blind docking calculation was employed to identify potential binding sites of PA on the GS structure. The 2D-QSAR model developed by us showed the importance of substituent 2 (C-7 position of PA) for the MTAase activity; hence, we have considered 7-NH-AMC (4), DAMC (6) and 7-AMC (13) as the model PA for the docking study. The resulting protein-ligand conformations for the model PA were found to be located on the surface region of the protein away from the known active site of Mtb GS. Figure 5 shows the representative binding modes of the best docked conformations for the three PA in the putative active site of Mtb GS. An important finding is that in all the docking poses obtained for DAMC, 7-AMC and 7-NH-AMC, a cation- $\pi$ interaction is observed between $\varepsilon-\mathrm{NH}_{3}$ group of Lys 4 and aromatic ring of coumarin (Figure 5). DAMC is found to form an additional 


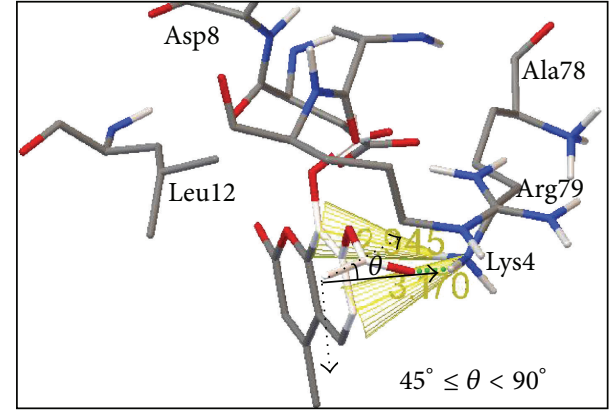

(a)

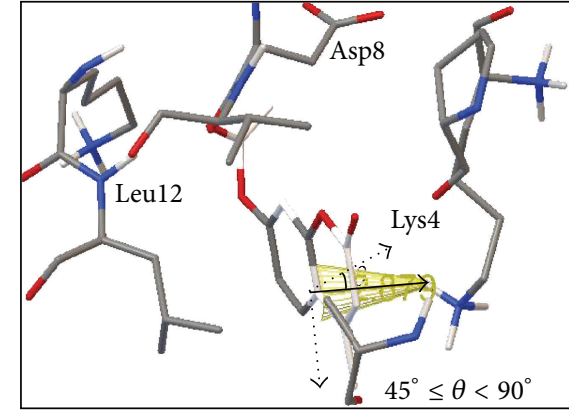

(b)

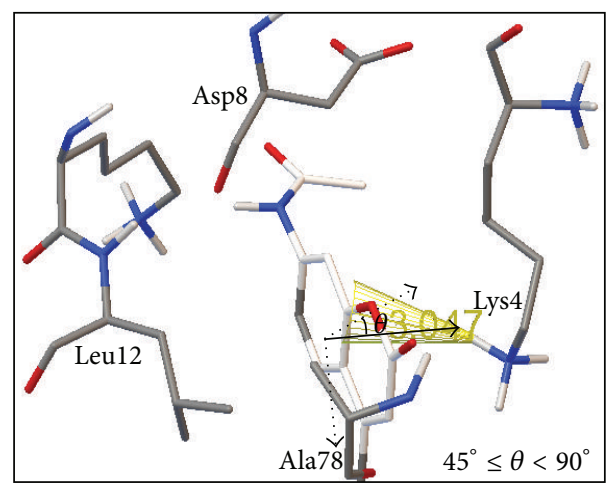

(c)

FIGURE 5: Cation- $\pi$ interaction (represented as yellow cone) between side chain of Lys4 of Mtb GS carrying net positive charge and aromatic rings of PA. (a) Simultaneous formation of H-bond (represented as green dotted line) is observed between $\varepsilon$ - NH2 group of Lys4 of Mtb GS and O-atom at C-7 position of DAMC; (b) interaction of 7-AMC with crystal structure of Mtb GS; (c) interaction of 7-NH-AMC with the crystal structure of Mtb GS. Cation- $\pi$ interaction occurs when the distance between a positively ionisable atom and the centroid of an aromatic ring is equal to or less than $4.0 \AA$, and the angle between the normal vector of the plane and the vector between the ionisable atom and the centroid is equal to or greater than $45^{\circ}$ and less than $90^{\circ}$ [30]. All the three interactions are in the permissible limits of the cation- $\pi$ interaction (as labeled in the figure).

H-bond between oxygen atom of C-7 acetyl group and $\varepsilon$ $\mathrm{NH}_{3}$ group of Lys4 (Figure 5(a)). The cation- $\pi$ interaction is a non-covalent interaction of a positively charged cation with $\pi$ electrons of an aromatic group. Experimental and $a b$ initio calculations indicated that this interaction is influenced by electrostatic forces between the monopole (cation) and the large quadrupole moment of the aromatic ring ( $\pi$-system) $[30,36]$. Cation- $\pi$ interactions involving the aromatic rings of ligand and amino acids with a net positive charge (Arg or Lys) have been reported to rationalize specific drug-receptor interactions [37-39]. Localization of ammonium-binding site in the crystal structure of GS from Salmonella typhimurium (PDB ID 2GLS) has implicated a cation- $\pi$ bonding between the Tyr179 and ammonium ion [40]. It is evident from the results that PAs interact with Mtb GS by way of cation- $\pi$ interaction, and such type of interaction may be conducive for the transfer of acetyl group to the receptor protein by Mtb GS. The observation that quadrupolar XX moment is one of the descriptor in the 2D-QSAR model very well validate the cation- $\pi$ interaction predicted by docking analysis for the Mtb GS-PA interaction.
3.3. ADMET Prediction. Most of drug failures at early and late pipeline occur due to undesired pharmacokinetics and toxicity problems. If these issues could be addressed early, it would be extremely advantageous for the drug discovery process. In view of these, the use of in silico methods to predict ADMET properties is intended as a first step in this direction to analyze the novel chemical entities to prevent wasting time on lead candidates that would be toxic or metabolized by the body into an inactive form and unable to cross membranes, and the results of such analysis are herein reported in Table 4 together with a biplot (Figure 6) and discussed. The pharmacokinetic profile of all the molecules under investigation was predicted by means of six precalculated ADMET models provided by the Discovery Studio 2.1 program. The biplot shows the two analogous $95 \%$ and $99 \%$ confidence ellipses corresponding to HIA and BBB models. PSA was shown to have an inverse relationship (with percent human intestinal absorption and thus cell wall permeability [41]. Though a relationship of PSA to permeability has been demonstrated, the models usually do not take into account the effects of other descriptors. The fluid mosaic model of cell membrane 

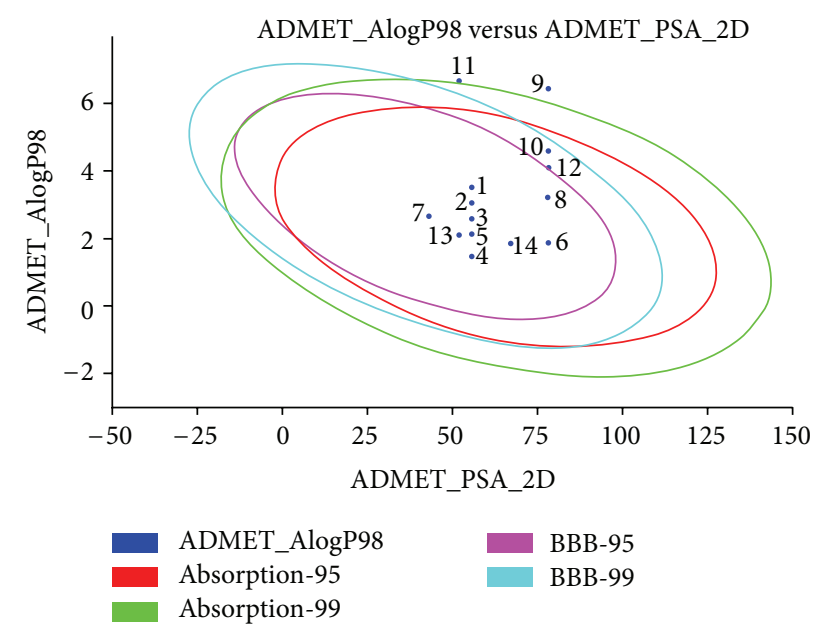

FIgURE 6: Prediction of drug absorption for various PA considered for anti-mycobacterial activity. Discovery Studio 2.1 (Accelrys, San Diego, CA) ADMET Descriptors, 2D polar surface area (PSA_2D) in $\AA^{2}$ for each compound is plotted against their corresponding calculated atom-type partition coefficient (ALogP98). The area encompassed by the ellipse is a prediction of good absorption with no violation of ADMET properties. On the basis of Egan et al. [19] absorption model the $95 \%$ and $99 \%$ confidence limit ellipses corresponding to the Blood Brain Barrier (BBB) and Intestinal Absorption models are indicated.

suggests that the membrane phospholipid bilayer is capable of hydrophobic and hydrophilic interactions, hence lipophilicity is also considered as a pivotal property for drug design. Lipophilicity could be assessed as the log of the partition coefficient between $n$-octanol and water $(\log \mathrm{P})$. Though $\log \mathrm{P}$ is generally used to estimate a compound's lipophilicity, the fact that $\log \mathrm{P}$ is a ratio raises a concern about the use of $\log \mathrm{P}$ to estimate hydrophilicity and hydrophobicity. Thus the information of $\mathrm{H}$-bonding characteristics as obtained by calculating PSA could be taken into consideration along with $\log \mathrm{P}$ calculation [19]. Therefore, a model with descriptors AlogP98 and PSA_2D with a bi-plot comprising 95\% and 99\% confidence ellipses was considered for the accurate prediction for the cell permeability of compounds. The $95 \%$ confidence, ellipse represents the region of chemical space where we can expect to find well-absorbed compounds ( $\geq 90 \%) 95$ out of 100 times. Whereas $99 \%$ is a confidence ellipse represents the region of chemical space with compounds having excellent absorption through cell membrane. According to the model for a compound to have an optimum cell permeability should follow the criteria (PSA $<140 \AA^{2}$ and AlogP98 < 5) [19]. All the compounds showed polar surface area (PSA) $<140 \AA^{2}$. Considering the AlogP98 criteria, all PAs had AlogP98 value $<5$, except compound 7 that has also in turn violated the $99 \%$ and $95 \%$ confidence ellipse for both HIA and BBB (Figure 6). Table 4 shows that majority of the compounds have low or undefined values for BBB penetration levels (levels 3 and 4 as mentioned in Table 2) with the exception of compound 7 having high value and compound $\mathbf{1 8}$ having medium BBB penetration level. The aqueous solubility plays a critical role in the bioavailability of the candidate drugs, and, with the exception of compound 7 having low aqueous solubility level (level 2) as referred in Table 2, all other PAs are having good or optimal aqueous solubility levels. Further, all compounds have been predicted to have hepatotoxicity level of 0 . The model was developed from available literature data of 382 compounds known to exhibit liver toxicity (i.e., positive dose-dependent hepatocellular, cholestatic, neoplastic, etc.) or trigger dose-related elevated aminotransferase levels in more than $10 \%$ of the human population [24]. The model classifies compounds either as "toxic" or "nontoxic" and provides a confidence level indicator of the likelihood of the models predictive accuracy (Table 2). Our results indicate that all PA are nontoxic to liver (level 0, Table 2), and thus they experience significant first-pass effect. Similarly, all ligands are satisfactory with respect to CYP2D6 liver (with reference to Table 2), suggesting that PA are noninhibitors of CYP2D6 (Table 4). This indicates that all PAs are well metabolized in Phase-I metabolism. Finally, the ADMET plasma protein binding property prediction denotes that all of 14 PAs with an exception of compounds 6 and 7 have binding $\geq 90 \%$ and $\geq 95 \%$, respectively, (refer to Table 2), clearly suggesting that most PAs have good bioavailability and are not likely to be highly bound to carrier proteins in the blood. An interesting observation was that the dihydroxy analogue of PA, that is, 7,8-dihydroxy-4-methylcoumarin (DHMC) (compound 14), which is the deacetylated product of MTAase activity, was also found to pass the entire ADMET test. This observation denotes that even by product of MTAase reaction is nontoxic.

\section{Conclusion}

We have made an effort to develop QSAR models using the kinetic constants and the MIC values to address the fact that TAase activity was leading to the antimycobacterial activity. The study indicated that Balaban index at C-7 position of PA was the only contributing descriptor for MTAase activity. The Balaban index, number of nitrogen atom at C-7 position of PA and quadrupole XX component (whole molecule), showed a good contribution to the antimycobacterial activity. Our observation of an overlapping descriptor (Balaban topological index at substituent 2) from our two QSAR models, thus clearly indicates that TAase activity mediated by GS utilizing PA as acetoxy group donor was leading to the antimycobacterial activity of PA. Further majority of PAs were found to have favorable ADMET characteristics. ADMET studies proved that PA can be developed as a potential antimycobacterial drug. The deacetylated product of TAase activity, DHMC, was also found to pass the entire ADMET test. An important finding is that in all the docking poses obtained for potent PA, a cation- $\pi$ interaction is observed between $\varepsilon-\mathrm{NH}_{3}$ group of Lys 4 and aromatic ring of coumarin. DAMC is found to form an additional H-bond between oxygen atom of C-7 acetyl group and $\varepsilon-\mathrm{NH} 3$ group of Lys4. Cation- $\pi$ interactions result essentially from a quadrupolar electrostatic interaction. The results of QSAR and docking studies validated each other and provided insight into the structural requirements for PA and Mtb GS interaction. 


\section{Abbreviations}

MTAase: Mycobacterial TAase

PA: $\quad$ Polyphenolic acetates

GS: Calreticulin glutamine synthetase

DAMC: $\quad$ 7,8-Diacetoxy-4-methylcoumarin

7-AMC: 7-acetoxy-4-methylcoumarin

7-NH-AMC: 7-NH-acetoxy-4-methylcoumarin

QSAR: Quantitative structure activity relationship

ADMET: Absorption distribution metabolism elimination toxicity

PSA: $\quad$ Polar surface area.

\section{Acknowledgments}

The financial assistance of the Department of Biotechnology Govt. of New Delhi, India is gratefully acknowledged. This research was partially supported by grants from the Ministry of Chemicals and Fertilizers, Government of India, India.

\section{References}

[1] H. G. Raj, V. S. Parmar, S. C. Jain et al., "Mechanism of biochemical action of substituted 4-methylbenzopyran-2-ones. Part 4: hyperbolic activation of rat liver microsomal nadphcytochrome $\mathrm{C}$ reductase by the novel acetylator 7,8-diacetoxy4-methylcoumarin," Bioorganic \& Medicinal Chemistry, vol. 7, no. 2, pp. 369-373, 1999.

[2] H. G. Raj, V. S. Parmar, S. C. Jain et al., "Mechanism of biochemical action of substituted 4-methylbenzopyran-2ones. Part 7: assay and characterization of 7,8-diacetoxy-4methylcoumarin:protein transacetylase from rat liver microsomes based on the irreversible inhibition of cytosolic glutathione S-Transferase," Bioorganic \& Medicinal Chemistry, vol. 8, no. 7, pp. 1707-1712, 2000.

[3] P. Khurana, R. Kumari, P. Vohra et al., "Acetoxy drug: protein transacetylase catalyzed activation of human platelet nitric oxide synthase by polyphenolic peracetates," Bioorganic \& Medicinal Chemistry, vol. 14, pp. 575-583, 2006.

[4] H. G. Raj, R. Kumari, S. Bansal et al., "Novel function of calreticulin: characterization of calreticulin as a transacetylasemediating protein acetylator independent of acetyl CoA using polyphenolic acetates "' Pure and Applied Chemistry, vol. 78, pp. 985-992, 2006.

[5] Seema, R. Kumari, G. Gupta et al., "Characterization of protein transacetylase from human placenta as a signaling molecule calreticulin using polyphenolic peracetates as the acetyl group donors," Cell Biochemistry and Biophysics, vol. 47, pp. 53-64, 2007.

[6] E. Kohli, M. Gaspari, H. G. Raj et al., "Acetoxy drug: protein transacetylase of buffalo liver-characterization and mass spectrometry of the acetylated protein product," Biochimica Et Biophysica Acta, vol. 1698, pp. 55-66, 2004.

[7] S. Bansal, M. Gaspari, H. G. Raj et al., "Calreticulin transacetylase mediates the acetylation of nitric oxide synthase by polyphenolic acetate," Applied Biochemistry and Biotechnology, vol. 144, pp. 37-45, 2008.

[8] G. Gupta, A. S. Baghel, S. Bansal et al., "Establishment of glutamine synthetase of Mycobacterium smegmatis as a protein acetyltransferase utilizing polyphenolic acetates as the acetyl group donors," Journal of Biochemistry, vol. 144, no. 6, pp. 709715, 2008.

[9] A. S. Baghel, R. Tandon, G. Gupta et al., "Characterization of protein acyltransferase function of recombinant purified GlnA1 from Mycobacterium tuberculosis: a moon lighting property," Microbiological Research, vol. 166, pp. 662-672, 2011.

[10] G. R. Hirschfield, M. McNeil, and P. J. Brennan, "Peptidoglycanassociated polypeptides of Mycobacterium tuberculosis," Journal of Bacteriology, vol. 172, no. 2, pp. 1005-1013, 1990.

[11] G. Harth, D. L. Clemens, M. A. Horwitz et al., "Glutamine synthetase of Mycobacterium tuberculosis: extracellular release and characterization of its enzymatic activity," Proceedings of the National Academy of Sciences of the United States of America, vol. 91, pp. 9342-9346, 1994.

[12] O. W. Griffith and A. Meister, "Differential inhibition of glutamine and $\gamma$-glutamylcysteine synthetases by $\alpha$-alkyl analogs of methionine sulfoximine that induce convulsions," Journal of Biological Chemistry, vol. 253, no. 7, pp. 2333-2338, 1978.

[13] B. Lejczak, H. Starzemska, and P. Mastalerz, "Inhibition of rat liver glutamine synthetase by phosphonic analogues of glutamic acid," Experientia, vol. 37, no. 5, pp. 461-462, 1981.

[14] R. Tandon, P. Ponnan, N. Aggarwal et al., "Characterization of 7-amino-4-methylcoumarin as an effective antitubercular agent: structure-activity relationships," Journal of Antimicrobial Chemotherapy, vol. 66, pp. 2543-2555, 2011.

[15] A. Kathuria, A. Gupta, N. Priya et al., "Specificities of calreticulin transacetylase to acetoxy derivatives of 3-alkyl-4methylcoumarins: effect on the activation of nitric oxide synthase," Bioorganic \& Medicinal Chemistry, vol. 17, pp. 1550-1556, 2009.

[16] Hyperchem Release8. Windows Molecular Modelling System, Hypercube, Inc. and Autodesk, Inc. Developed by Hypercube, Inc.

[17] A. Golbraikh and A. Tropsha, "Beware of q2!", Journal of Molecular Graphics and Modelling, vol. 20, no. 4, pp. 269-276, 2002.

[18] A. Tropsha, P. Gramatica, and V. K. Gombar, "The importance of being earnest: validation is the absolute essential for successful application and interpretation of QSPR models," QSAR and Combinatorial Science, vol. 22, no. 1, pp. 69-77, 2003.

[19] W. J. Egan, K. M. Merz, and J. J. Baldwin, "Prediction of drug absorption using multivariate statistics," Journal of Medicinal Chemistry, vol. 43, no. 21, pp. 3867-3877, 2000.

[20] A. Cheng and K. M. Merz, "Prediction of aqueous solubility of a diverse set of compounds using quantitative structure-property relationships," Journal of Medicinal Chemistry, vol. 46, no. 17, pp. 3572-3580, 2003.

[21] W. J. Egan and G. Lauri, "Prediction of intestinal permeability," Advanced Drug Delivery Reviews, vol. 54, no. 3, pp. 273-289, 2002.

[22] S. L. Dixon and K. M. Merz, "One-dimensional molecular representations and similarity calculations: methodology and validation," Journal of Medicinal Chemistry, vol. 44, no. 23, pp. 3795-3809, 2001.

[23] R. G. Susnow and S. L. Dixon, "Use of robust classification techniques for the prediction of human cytochrome P450 2D6 inhibition," Journal of Chemical Information and Computer Sciences, vol. 43, pp. 1308-1315, 2003.

[24] A. Cheng and S. L. Dixon, "In silico models for the prediction of dose-dependent human hepatotoxicity," Journal of ComputerAided Molecular Design, vol. 17, no. 12, pp. 811-823, 2003. 
[25] C. Hetényi and D. Spoelvander, "Efficient docking of peptides to proteins without prior knowledge of the binding site," Protein Science, vol. 11, pp. 1729-1737, 2002.

[26] G. M. Morris, D. S. Goodsell, R. S. Halliday et al., "Automated docking using a Lamarckian genetic algorithm and an empirical binding free energy function," Journal of Computational Chemistry, vol. 19, no. 14, pp. 1639-1662, 1998.

[27] W. W. Krajewski, A. T. Jones, S. L. Mowbray et al., "Structure of Mycobacterium tuberculosis glutamine synthetase in complex with a transition-state mimic provides functional insights," Proceedings of the National Academy of Sciences of the United States of America, vol. 102, pp. 10499-10504, 2005.

[28] M. F. Sanner, B. S. Duncan, C. J. Carrillo et al., "Proteinmorphosis: a mechanical model for protein conformational changes," in Proceedings of the Pacific Symposium in Biocomputing (PSB '99), pp. 401-412, Big Island, Hawaii, USA, 1999.

[29] T. J. A. Ewing and I. D. Kuntz, "Critical evaluation of search algorithms for automated molecular docking and database screening," Journal of Computational Chemistry, vol. 18, no. 9, pp. 1175-1189, 1997.

[30] D. A. Dougherty, "Cation- $\pi$ interactions in chemistry and biology: a new view of benzene, Phe, Tyr, and Trp," Science, vol. 271, no. 5246, pp. 163-168, 1996.

[31] A. T. Balaban, "Highly discriminating distance-based topological index," Chemical Physics Letters, vol. 89, pp. 399-404, 1982.

[32] D. Mandloi, S. Joshi, P. V. Khadikar et al., "QSAR study on the antibacterial activity of some sulfa drugs: building blockers of Mannich bases," Bioorganic \& Medicinal Chemistry Letters, vol. 15, pp. 405-411, 2005.

[33] S. C. Basak, D. P. Gieschen, D. K. Harriss, and V. R. Magnuson, "Physicochemical and topological correlates of the enzymatic acetyltransfer reaction," Journal of Pharmaceutical Sciences, vol. 72, no. 8, pp. 934-937, 1983.

[34] P. Singh, P. Ponnan, S. Krishnan et al., "Protein acyltransferase function of purified calreticulin. Part 1: characterization of propionylation of protein utilizing propoxycoumarin as the propionyl group donor," Journal of Biochemistry, vol. 147, no. 5, pp. 625-632, 2010.

[35] Y. Chen, R. Sprung, Y. Tang et al., "Lysine propionylation and butyrylation are novel post-translational modifications in histones," Molecular \& Cellular Proteomics, vol. 6, pp. 812-819, 2007.

[36] J. H. Williams, "The molecular electric quadrupole moment and solid-state architecture," Accounts of Chemical Research, vol. 26, pp. 593-598, 1993.

[37] M. Dennis, J. Giraudat, F. Kotzyba-Hibert et al., "Amino acids of the torpedo marmorata acetylcholine receptor $\alpha$ subunit labeled by a photoaffinity ligand for the acetylcholine binding site," Biochemistry, vol. 27, no. 7, pp. 2346-2357, 1988.

[38] P. D. Leeson, R. Baker, R. W. Carling et al., "Amino acid bioisosteres: design of 2-quinolone derivatives as glycine-site $\mathrm{N}$ methyl-D-aspartate receptor antagonists," Bioorganic \& Medicinal Chemistry Letters, vol. 3, pp. 299-304, 1993.

[39] B. Yang, J. Wright, M. E. Eldefrawi, S. Pou, and A. D. MacKerell, "Conformational, aqueous solvation, and $\mathrm{pK}(\mathrm{a})$ contributions to the binding and activity of cocaine, WIN 32065-2, and the WIN vinyl analog," Journal of the American Chemical Society, vol. 116, no. 19, pp. 8722-8732, 1994.

[40] S. H. Liaw, I. Kuo, and D. Eisenberg, "Discovery of the ammonium substrate site on glutamine synthetase, a third cation binding site," Protein Science, vol. 4, no. 11, pp. 2358-2365, 1995.
[41] K. Palm, P. Stenberg, K. Luthman, and P. Artursson, "Polar molecular surface properties predict the intestinal absorption of drugs in humans," Pharmaceutical Research, vol. 14, no. 5, pp. 568-571, 1997. 

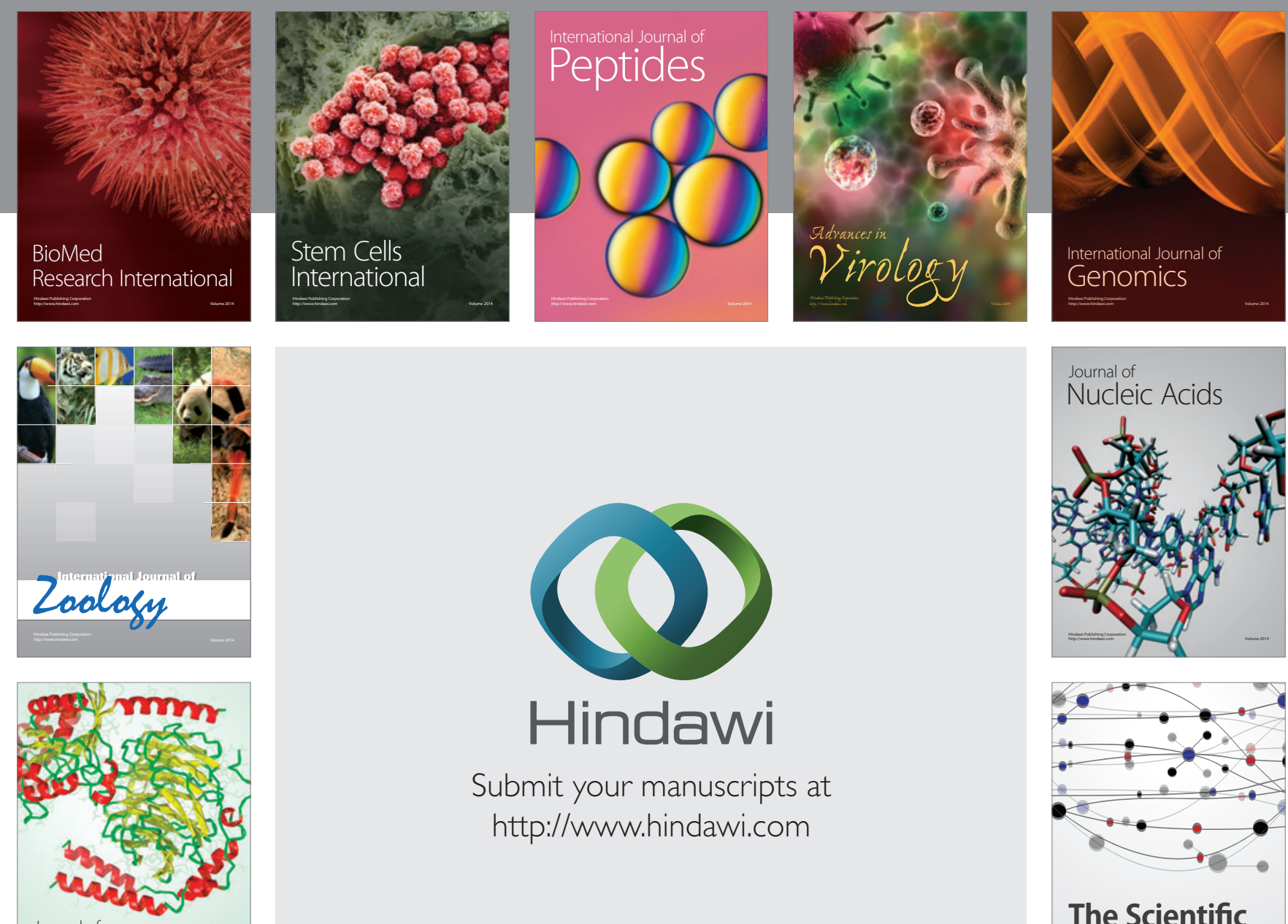

Submit your manuscripts at

http://www.hindawi.com

Journal of
Signal Transduction
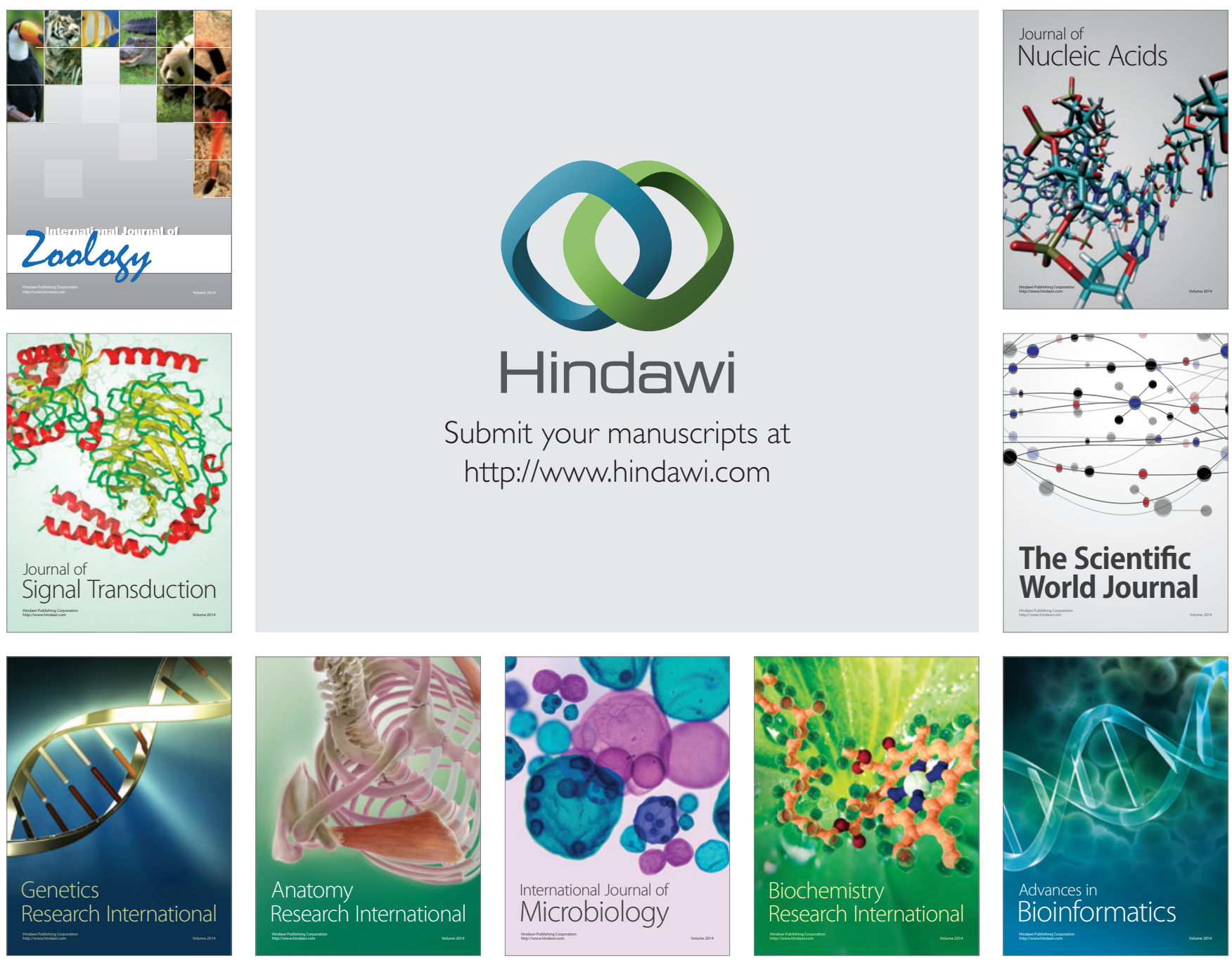

The Scientific World Journal
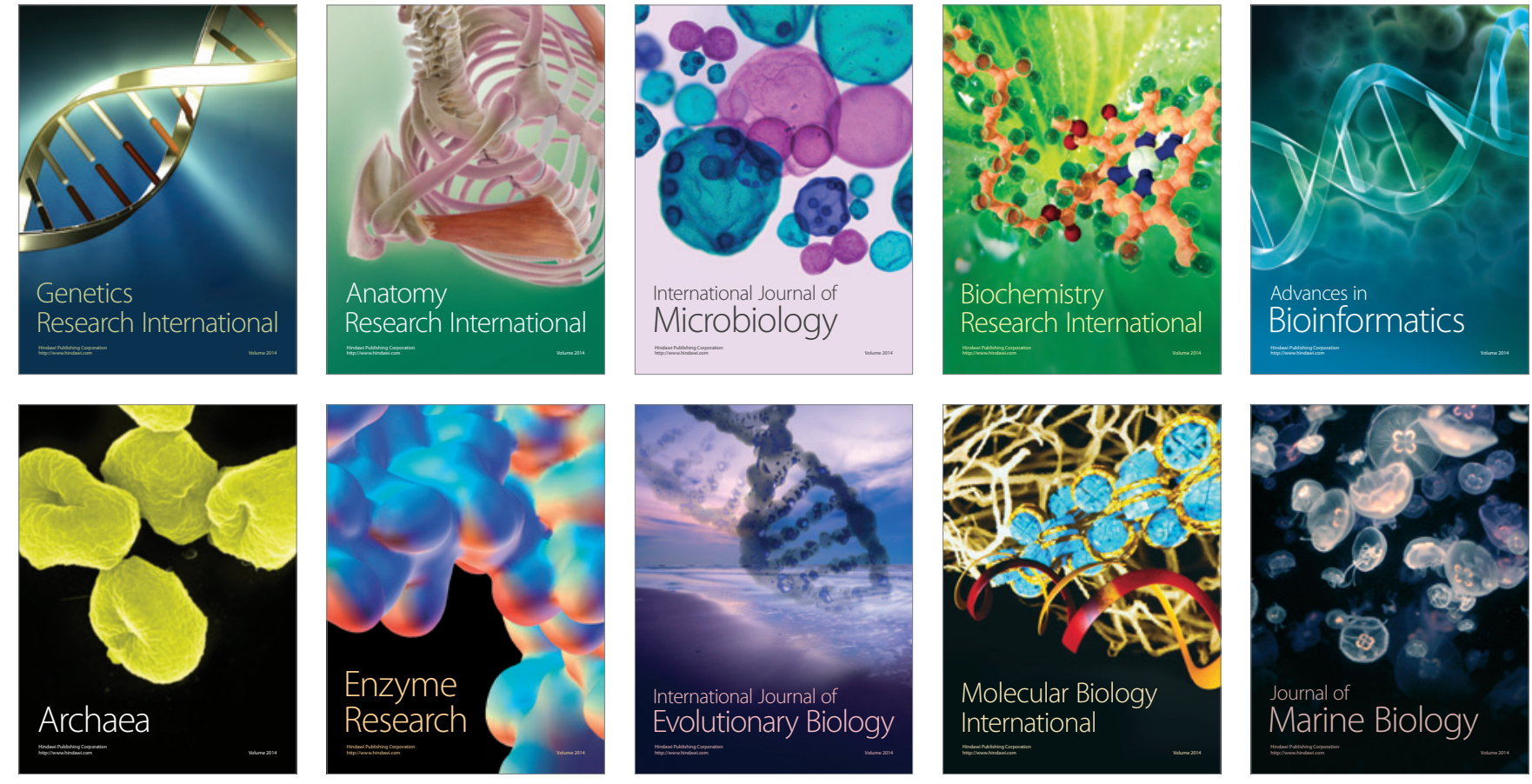